\title{
Hyper-Kähler geometry and semi-geostrophic theory
}

\author{
By Sylvain Delahaies \& Ian Roulstone \\ Department of Mathematics, University of Surrey, Guildford, GU2 7XH UK
}

We use the formalism of Monge-Ampère operators to study the geometric properties of the Monge-Ampère equations arising in semi-geostrophic theory and related models of geophysical fluid dynamics. We show how Kähler and hyper-Kähler structures arise, and the Legendre duality arising in semi-geostrophic theory is generalized to other models of nearly geostrophic flows.

Keywords: Semi-geostrophic theory, Balanced models, Monge-Ampère equations, hyper-Kähler geometry

\section{Introduction}

Semi-geostrophic (SG) theory is an approximation to the Navier-Stokes-based equations for atmosphere-ocean dynamics, which has proven especially useful in the study of weather fronts, land/sea breezes, flow over orography, monsoons, and largescale ocean currents. The utility of SG theory is a consequence of several elegant mathematical properties, which include hamiltonian structure, contact (Legendre) duality, and the ubiquity of a Monge-Ampère equation. The latter is the second order nonlinear partial differential equation that, in the context of SG theory, relates the potential vorticity to the wind and temperature fields. Contact transformations of the Monge-Ampère equation allow the $\mathrm{SG}$ equations to be solved analytically for certain idealised flows; see, for example, Blumen (1981), Hoskins (1975) and Shutts (1991). The Legendre duality can be used to construct novel numerical techniques, including finite element methods, which have proven especially useful in exploiting the lagrangian formulation of the equations (Cullen \& Purser (1984, 1989)). SG theory is one of many so-called 'balanced models' (balance in this context refers to the geostrophic balance between fluid velocity and pressure gradient in flows on a rotating domain), although SG theory retains a special significance because of its elegant geometrical properties. Roubtsov \& Roulstone $(1997,2001)$ showed that a hierachy of balanced models possess symplectic, contact and Monge-Ampère structures akin to SG theory, and that these hitherto apparently disconnected features can be viewed as the component parts of a hyper-Kähler structure. However, it was believed that SG itself could not be formulated in terms of hyper-Kähler geometry, and several other questions concerning the relevance of hyper-Kähler structures to balanced models remained open. For an extensive discussion of these issues, see McIntyre \& Roulstone (2002).

The purpose of this paper is to address these issues. The approach adopted by Roubtsov \& Roulstone $(1997,2001)$ was based on the special significance attached to the jacobian of the map between local symplectic coordinates and the 
lagrangian configuration coordinates of the fluid $\dagger$. In this paper we adopt a different approach. Instead of focussing on the map between local symplectic coordinates and the lagrangian fluid coordinates, we apply the methods of Kushner et al. (2007) and re-derive the geometric properties using the theory of Monge-Ampère operators. The salient difference between the two approaches may be summarized as follows: Roubtsov \& Roulstone $(1997,2001)$ study the geometry associated with $\mathcal{D}(\phi)$, where $\mathcal{D}$ denotes the determinant of the hessian matrix of a dependent variable $\phi$ (this determinant is the jacobian discussed above), whereas we study the Monge-Ampère equation $\mathcal{D}(\phi)=q$, where $q$ is a given function of the independent variables. As a consequence, we are able to show that SG theory does indeed possess a hyper-Kähler structure, and the issues raised by McIntyre \& Roulstone (2002) can be resolved.

Although a thermal structure is crucial for the formation of phenomena such as fronts and sea breezes, the salient mathematical features of SG theory can be studied within the context of the shallow water equations (Roulstone \& Sewell (1997), McIntyre \& Roulstone (2002)), which is the approach we adopt in this paper. The paper is organised as follows: in Section 2, after reviewing SG theory and its essential mathematical features, we discuss the Hamiltonian balanced models (HBMs) derived in McIntyre \& Roulstone op. cit. In Section 3, we introduce hyperKähler structures and we show how the phase space of HBMs is endowed with such structures. In Section 4, we follow the approach of Kushner et al. (2007) to study the Monge-Ampère equation relating the geopotential to the absolute vorticity from a geometric perspective; we derive a new example of a hyper-Kähler structure, extending the hyper-Kähler structure described in Section 3 for HBMs, which incorporates SG theory. In Section 5 we use this new example to extend the Legendre duality arising in SG theory to HBMs.

\section{Balanced models}

\section{(a) Semi-geostrophic theory}

In the semi-geostrophic regime, the motion of a shallow layer of inviscid fluid of depth $\eta(x, y, t)$, rotating with constant angular frequency $f / 2$, can be approximated by replacing the acceleration $\dot{\mathbf{u}}$ in the momentum equations with the Lagrangian time derivative of the geostrophic wind:

$$
\dot{\mathbf{u}}_{\mathrm{g}}+f \mathbf{k} \times \dot{\mathbf{x}}+g \nabla \eta=0
$$

Here $\mathbf{x}=(x, y)^{\mathrm{T}} \in \mathbb{R}^{2}$ denotes the positions of the fluid particles, $g$ is a constant representing the acceleration due to gravity, $\mathbf{k}$ is the unit vertical vector, and we assume no bottom topography. Throughout this paper $f$, which denotes the Coriolis parameter, is assumed to be a constant. The geostrophic wind $\mathbf{u}_{\mathrm{g}}=\left(u_{\mathrm{g}}, v_{\mathrm{g}}\right)^{\mathrm{T}}$ is defined by

$$
u_{\mathrm{g}}=-f \frac{\partial \phi}{\partial y}, v_{\mathrm{g}}=f \frac{\partial \phi}{\partial x}
$$

$\dagger$ The singularities of the map between these coordinate systems is precisely the feature that, via Legendre duality, can be interpreted in the context of fronts (Chynoweth \& Sewell (1989)). 
with $\phi$ denoting the geopotential $\phi=g \eta / f^{2}$. The positions $\mathbf{x}$ are functions of the Lagrangian mass coordinates $a$ and $b$, and the time $t$ :

$$
x=x(a, b, t), y=y(a, b, t),
$$

where Lagrangian mass coordinates are chosen so that $a=x(a, b, 0), b=y(a, b, 0)$. The superposed dot denotes the Lagrangian time derivative following a particle, that is $\partial / \partial t$ with $a$ and $b$ held fixed. The incompressibility hypothesis requires $\eta$ to satisfy the relation $\eta_{0} \mathrm{~d} a \mathrm{~d} b=\eta(x, y, t) \mathrm{d} x \mathrm{~d} y$, where $\eta_{0}$ is a constant initial state. If we further assume that $\eta_{0}=1$, then the height field $\eta$ is given by

$$
\eta(x, y, t)=\frac{\partial(a, b)}{\partial(x, y)} .
$$

This equation provides us with an implicit form of the continuity equation. The momentum equations (2.1) together with the continuity equation (2.3) constitute the shallow-water version of the SG equations. Using (2.1) and taking the Lagrangian time derivative of $(2.3)$, we can show that the potential vorticity $\mathcal{Q}_{\text {sg }}$ defined by

$$
\mathcal{Q}_{\mathrm{sg}}=\frac{g}{f \phi}\left[1+\frac{\partial^{2} \phi}{\partial x^{2}}+\frac{\partial^{2} \phi}{\partial y^{2}}+\frac{\partial^{2} \phi}{\partial x^{2}} \frac{\partial^{2} \phi}{\partial y^{2}}-\left(\frac{\partial^{2} \phi}{\partial x \partial y}\right)^{2}\right],
$$

is conserved, i.e. $\dot{\mathcal{Q}}_{\mathrm{sg}}=0$. Equation (2.4) is a Monge-Ampère type equation for $\phi$, given $\mathcal{Q}_{\text {sg }}$ and appropriate boundary conditions. This equation is elliptic when $\mathcal{Q}_{\text {sg }}>0$.

Hoskins (1975) showed that integration of the SG equations is facilitated by the use of the coordinate transformation $\mathbf{x} \mapsto \mathbf{X}=(X, Y)$ given by

$$
X=x+\frac{\partial \phi}{\partial x} \quad, \quad Y=y+\frac{\partial \phi}{\partial y},
$$

in terms of which the potential vorticity (2.4) can then be expressed in jacobian form

$$
\mathcal{Q}_{\mathrm{sg}}=\frac{g}{f \phi} \frac{\partial(X, Y)}{\partial(x, y)} \text {. }
$$

The coordinates $(X, Y)$ are often referred to as geostrophic coordinates because, using the definition (2.5), we can express (2.1) as $\dot{\mathbf{X}}=\mathbf{u}_{\mathrm{g}}$; that is, the motion in these coordinates is exactly geostrophic. The so-called geostrophic coordinate transformation $\mathbf{x} \mapsto \mathbf{X}$ can be interpreted in terms of Legendre duality (Chynoweth $\&$ Sewell (1989)). That is, given the two dual functions $\mathcal{P}(x, y, t)$ and $\mathcal{R}(X, Y, t)$ defined by

$$
\begin{aligned}
\mathcal{P}(x, y, t) & =\phi(x, y, t)+\frac{1}{2}\left(x^{2}+y^{2}\right), \\
\mathcal{R}(X, Y, t) & =x X+y Y-\mathcal{P},
\end{aligned}
$$

we see that the transformation $(x, y) \mapsto(X, Y)$ is a Legendre transformation, because using $(2.7),(2.8)$ and $(2.5)$, we have

$$
\frac{\partial \mathcal{P}}{\partial x}=X, \frac{\partial \mathcal{P}}{\partial y}=Y, \text { and the dual relations } \frac{\partial \mathcal{R}}{\partial X}=x, \frac{\partial \mathcal{R}}{\partial Y}=y .
$$


We define a dual potential $\Phi$, referred to as the Bernoulli potential, as the function

$$
\Phi(X, Y, t)=-\mathcal{R}+\frac{1}{2}\left(X^{2}+Y^{2}\right)=\phi(x, y, t)+\frac{1}{2}\left[\left(\frac{\partial \phi}{\partial x}\right)^{2}+\left(\frac{\partial \phi}{\partial y}\right)^{2}\right]
$$

Using (2.9) we can show that

$$
\frac{\partial \phi}{\partial x}=\frac{\partial \Phi}{\partial X}, \frac{\partial \phi}{\partial y}=\frac{\partial \Phi}{\partial Y}
$$

This property, referred to by Roulstone \& Sewell (1997) as the gradient transformation property of the geostrophic coordinate transformation, allows us to write the momentum equations (2.1) in the Hamiltonian form

$$
\dot{X}=-f \frac{\partial \Phi}{\partial Y}, \dot{Y}=f \frac{\partial \Phi}{\partial X} .
$$

Finally, as first pointed out by Blumen (1981), the transformation

$$
\left(x, y, \phi, \frac{\partial \phi}{\partial x}, \frac{\partial \phi}{\partial y}\right) \mapsto\left(X, Y, \Phi, \frac{\partial \Phi}{\partial X}, \frac{\partial \Phi}{\partial Y}\right)
$$

defines a strict contact transformation: using (2.5), (2.10) and (2.11) we see that

$$
\mathrm{d} \Phi-\frac{\partial \Phi}{\partial X} \mathrm{~d} X-\frac{\partial \Phi}{\partial Y} \mathrm{~d} Y=\mathrm{d} \phi-\frac{\partial \phi}{\partial x} \mathrm{~d} x-\frac{\partial \phi}{\partial y} \mathrm{~d} y .
$$

See McIntyre \& Roulstone $(2002, \S 5)$, for further details.

\section{(b) More accurate balanced models}

Despite its remarkable conceptual simplicity and its useful mathematical properties, the SG approximation is formally correct only to leading order in Rossby number (see, e.g. McIntyre \& Roulstone (2002), for discussion). Therefore the question of how to derive more accurate models that would retain the essential features of SG theory has been much studied since its introduction in the mid-seventies. Using the framework of constrained Hamiltonian dynamics, pioneered by Salmon (1983, 1985) and by Allen \& Holm (1996 \& references therein), McIntyre \& Roulstone (1996) attempt to provide an answer to this question by systematically deriving a class of Hamiltonian balanced models. These models conserve the potential vorticity, $\mathcal{Q}_{c}$, defined by

$$
\mathcal{Q}_{c}=\frac{g}{f \phi}\left[1+\frac{\partial^{2} \phi}{\partial x^{2}}+\frac{\partial^{2} \phi}{\partial y^{2}}+\left(1-c^{2}\right)\left(\frac{\partial^{2} \phi}{\partial x^{2}} \frac{\partial^{2} \phi}{\partial y^{2}}-\left(\frac{\partial^{2} \phi}{\partial x \partial y}\right)^{2}\right)\right],
$$

where $c \in \mathbb{R}$. Different values of $c$ define different balanced models. For example, when $c=0, \mathcal{Q}_{\mathrm{c}}$ corresponds to $(2.4)$ - the functional form of the potential vorticity conserved by the semi-geostrophic equations. When $c=1$, the nonlinear term is eliminated in (2.13), and this is the conserved potential vorticity of Salmon's $L_{1}$ dynamics (Salmon (1985)). A third value of $c$ is of particular interest: when $c=\sqrt{3}$ equation (2.13) agrees to order two with an asymptotic expansion in terms 
of the Rossby number of the absolute vorticity for shallow-water dynamics. By comparison, $L_{1}$-dynamics $(c=1)$ is only accurate to order one and SG theory $(c=0)$ introduces the correct second order term but with the wrong coefficient ( +1 instead of -2 : see Snyder et al. (1991) for further discussion; also Delahaies (2009) §2.2.4). The so-called $\sqrt{3}$-model is the most accurate model within the class of models derived by McIntyre \& Roulstone.

McIntyre \& Roulstone (1996) showed that

$$
\mathbf{X}=(X, Y)^{\mathrm{T}}=\mathbf{x}+\nabla \phi-\mathrm{i} c \mathbf{k} \times \nabla \phi,
$$

where $\mathrm{i}=\sqrt{-1}$, are canonical coordinates for the Hamiltonian models. Note that $(X, Y)$ was used earlier to denote the geostrophic coordinates: we adopt the same notation since setting $c=0$ in (2.14) we obtain (2.5). As is the case of SG, the potential vorticity $\mathcal{Q}_{c}$ can be expressed in terms of the jacobian of the coordinate transformation

$$
\mathcal{Q}_{\mathrm{c}}=\frac{g}{f \phi} \frac{\partial(X, Y)}{\partial(x, y)} .
$$

Roubtsov and Roulstone op.cit. introduced a dual complex potential $\Phi$ given by

$$
\Phi(X, \bar{Y}, t)=\phi(x, y, t)+\frac{1}{2}\left[\left(\frac{\partial \phi}{\partial x}\right)^{2}+\left(\frac{\partial \phi}{\partial y}\right)^{2}\right]+\mathrm{i} c \frac{\partial \phi}{\partial x} \frac{\partial \phi}{\partial y}
$$

which satisfies

$$
\frac{\partial \Phi}{\partial \bar{X}}=0, \frac{\partial \Phi}{\partial Y}=0, \frac{\partial \Phi}{\partial X}=\frac{\partial \phi}{\partial x}, \frac{\partial \Phi}{\partial \bar{Y}}=\frac{\partial \phi}{\partial y},
$$

where the overbar is used to denote the complex congugate. The first two equations $\left((2.17)_{1}\right.$ and $\left.(2.17)_{2}\right)$ show that $\Phi$ is holomorphic in $X$ and $\bar{Y}$, and the last two equations $\left((2.17)_{3}\right.$ and $\left.(2.17)_{4}\right)$ are reminiscent of the gradient transformation property of SG (cf. (2.11)). Introducing the space spanned by the coordinate system $(X, \bar{Y}, \Phi, \partial \Phi / \partial X, \partial \Phi / \partial \bar{Y})$, endowed with the contact form $\mathrm{d} \Phi-(\partial \Phi / \partial X) \mathrm{d} X-$ $(\partial \Phi / \partial \bar{Y}) \mathrm{d} \bar{Y}$ - referred to in Roulstone \& Roubtsov (2001) as the semi-holomorphic contact bundle corresponding to the coordinates $(X, \bar{Y})$ - the transformation

$$
\left(x, y, \phi, \frac{\partial \phi}{\partial x}, \frac{\partial \phi}{\partial y}\right) \mapsto\left(X, \bar{Y}, \Phi, \frac{\partial \Phi}{\partial X}, \frac{\partial \Phi}{\partial \bar{Y}}\right)
$$

defines a strict contact transformation for, using (2.14), (2.16) and (2.17), we have

$$
\mathrm{d} \Phi-\frac{\partial \Phi}{\partial X} \mathrm{~d} X-\frac{\partial \Phi}{\partial \bar{Y}} \mathrm{~d} \bar{Y}=\mathrm{d} \phi-\frac{\partial \phi}{\partial x} \mathrm{~d} x-\frac{\partial \phi}{\partial x} \mathrm{~d} y
$$

Note that $\Phi$ was used earlier to denote the Bernoulli potential (2.10): again we adopt the same notation since setting $c=0$ the coordinate $X$ and $\bar{Y}$ are nothing but the geostrophic coordinates and (2.16) reduces (2.10). Just as for SG, the dynamics of HBMs can be formulated in terms of $X, \bar{Y}, \Phi, \partial \Phi / \partial X, \partial \Phi / \partial \bar{Y}, \dot{X}, \dot{\bar{Y}}$, however as stated in McIntyre \& Roulstone (2002) "there is no reason to expect $\Phi$ to enter into the evolution equations with anything like the simplicity of (2.12)".

In the following sections we introduce the geometry that explains why complex coordinates arise naturally in the jacobian of (2.15), and in turn this explains why SG theory does not possess complex structure. Then, by introducing the theory of Monge-Ampère operators, which associates geometrical structures to the equation (2.15), we derive new complex coordinates that incorporate SG theory. 


\section{Kähler and hyper-Kähler geometry}

The discovery of complex canonical coordinates for HBMs (McIntyre \& Roulstone (1996)) brings a new geometric framework in which to study these models (Roubtsov \& Roulstone $(1997,2001))$. We now introduce some notation and concepts of differential geometry, not all of which have appeared in our previous papers on this subject, that we shall require in subsequent sections. Further details can be found in Kushner et al. (2007) and McDuff \& Salamon (1998)

\section{(a) Definitions and properties}

Let $M$ be a $2 n$-dimensional manifold. An almost-complex structure $I$ on $M$ is a field of endomorphisms of the tangent bundle $T M$ such that $I^{2}=-\mathbf{1}_{2 n}$, where $\mathbf{1}_{2 n}$ denotes the $2 n \times 2 n$ identity matrix. The pair $(M, I)$ is called an almost-complex manifold. A map $F$ between two almost-complex manifolds $(M, I)$ and $\left(M^{\prime}, I^{\prime}\right)$ is said to be $\left(I, I^{\prime}\right)$-holomorphic if and only if for all $m \in M$ the tangent map $\mathrm{d} F_{m}$ is complex linear; that is $\mathrm{d} F_{m} \circ I(m)=I^{\prime}(F(m)) \circ \mathrm{d} F_{m}$. The almost-complex-structure $I$ is integrable if at any point $m \in M$ there exists a neighbourhood $\mathcal{U}_{m}$ of $m$ such that we can define $(I, \mathrm{i})$-holomorphic coordinates $z^{l}: \mathcal{U}_{m} \rightarrow \mathbb{C}, l=1 . . n$, in which $I$ takes the form

$$
I=\left(\begin{array}{cc}
\mathrm{i} \mathbf{1}_{n} & \mathbf{0} \\
\mathbf{0} & -\mathrm{i} \mathbf{1}_{n}
\end{array}\right)
$$

where $\mathbf{0}$ denotes the $n \times n$ zero matrix. This allows the identification of the manifold $M$ as a complex manifold: in the coordinate system $\left\{z^{1}, \ldots, z^{n}, \bar{z}^{1}, \ldots, \bar{z}^{n}\right\}$, where the overbar denotes the complex conjugate, operating with $I$ is equivalent to transforming $\mathrm{d} z^{l}$ into $\mathrm{id} z^{l}$ and $\mathrm{d} \bar{z}^{l}$ into $-\mathrm{id} \bar{z}^{l}$. When $I$ is integrable it is called a complex structure, and we will say that a coordinate system $\left(z^{l}, \bar{z}^{l}\right)$, that realizes the identification of the manifold $M$ as a complex manifold, is an adapted coordinate system for $I$, or that it is induced by the complex structure $I$.

A Riemannian metric $h$ together with an almost-complex structure $I$, and a nondegenerate 2-form $\varpi$ satisfying the compatibility condition $\varpi(\cdot, \cdot)=h(I \cdot, \cdot)$, define an almost-hermitian structure on $M$. When $\varpi$ is closed this structure is called almost-Kähler; in addition, if $I$ is integrable this structure defines a Kähler structure on $M$. The metric $h$ is then called a Kähler metric and $\varpi$ a Kähler form. Note that any two objects of the triple $(h, I, \varpi)$ determine the third one from the compatibility condition.

Assume now that $M$ is a $4 n$-dimensional manifold and let $h$ be a Riemannian metric on $M$. A hyper-Kähler structure on $(M, h)$ is prescribed by a triple of linearly independent complex structures $\left(I_{1}, I_{2}, I_{3}\right)$ satisfying the quaternion relations

$$
I_{1}^{2}=I_{2}^{2}=I_{3}^{2}=-\mathbf{1}_{4 n}, I_{1} I_{2} I_{3}=-\mathbf{1}_{4 n},
$$

and such that $h$ is Kähler with respect to all three complex structures. Alternatively, the hyper-Kähler structure can be prescribed by the triple of symplectic 2-forms $\left(\omega_{1}, \omega_{2}, \omega_{3}\right)$ defined by $\omega_{i}(\cdot, \cdot)=h\left(I_{i} \cdot, \cdot\right), i=1,2,3$. When endowed with a hyperKähler structure, the Riemannian manifold $(M, h)$ is called a hyper-Kähler manifold and $h$ is said to define a hyper-Kähler metric.

Let us consider a simple example which will prove useful in the subsequent sections to describe the geometric structures arising in HBMs and SG theory: take 
$\mathbb{C}^{2}$ endowed with the coordinate system $\{X, Y, \bar{X}, \bar{Y}\}$. Then $\mathbb{C}^{2}$ is endowed with a natural Kähler structure $(I, h)$, where $I$ is the canonical complex structure on $\mathbb{C}^{2}$ given by equation (3.1) and $h$ is the standard complex metric given by

$$
h=\mathrm{d} X \otimes \mathrm{d} \bar{X}+\mathrm{d} Y \otimes \mathrm{d} \bar{Y} .
$$

Then the Kähler 2-form is given by

$$
\varpi=-\frac{\mathrm{i}}{2}(\mathrm{~d} X \wedge \mathrm{d} \bar{X}+\mathrm{d} Y \wedge \mathrm{d} \bar{Y}) .
$$

This example will be referred to as the Kähler structure induced by the coordinate system $(X, Y)$. We further obtain the hyper-Kähler structure induced by the coordinate system $(X, Y)$ by considering the standard complex metric $h$ together with the triple of 2 -forms $\left(\omega_{1}, \omega_{2}, \omega_{3}\right)$ given by

$$
\omega_{1}=\operatorname{Re} \mathrm{d} X \wedge \mathrm{d} Y, \omega_{2}=-\frac{\mathrm{i}}{2}(\mathrm{~d} X \wedge \mathrm{d} \bar{X}+\mathrm{d} Y \wedge \mathrm{d} \bar{Y}), \omega_{3}=\operatorname{Im} \mathrm{d} X \wedge \mathrm{d} Y .
$$

In this example the hyper-Kähler structure is expressed relative to the Kähler structure defined by $\left(h, \omega_{2}\right)$. By definition a hyper-Kähler manifold $M$ is Kähler with respect to all three complex structures; then at any point $m \in M$ there exists a neighborhood in which three complex coordinate systems coexist, each of them providing an identification of $M$ as a complex manifold.

(b) Hyper-Kähler geometry \& balanced models: canonical coordinates

The coordinate system $\mathbf{X}=(X, Y)^{\mathrm{T}}$ given by $(2.14)$

$$
X=x+p+\mathrm{i} c q, Y=y+q-\mathrm{i} c p,
$$

where $(p, q)=(\partial \phi / \partial x, \partial \phi / \partial y)$, defines an adapted coordinate system for a complex structure on the cotangent bundle $T^{*} \mathbb{R}^{2}$. The Kähler structure $(h, \varpi)$ induced on $T^{*} \mathbb{R}^{2}$ by $\mathbf{X}$ is given, in terms of the coordinate system $\{x, y, p, q\}$, by

$$
h=\mathrm{d} x \otimes \mathrm{d} x+\mathrm{d} y \otimes \mathrm{d} y+\mathrm{d} x \otimes \mathrm{d} p+\mathrm{d} y \otimes \mathrm{d} q+\left(1+c^{2}\right)(\mathrm{d} p \otimes \mathrm{d} p+\mathrm{d} q \otimes \mathrm{d} q),
$$

and

$$
\varpi=c(\mathrm{~d} x \wedge \mathrm{d} q+\mathrm{d} p \wedge \mathrm{d} y+2 \mathrm{~d} p \wedge \mathrm{d} q) .
$$

Using the relation $\varpi(\cdot, \cdot)=h\left(I_{\omega}, \cdot\right)$, the complex structure $I_{\omega}$ is given by

$$
I_{\omega}=\frac{1}{c}\left(\begin{array}{cccc}
0 & -1 & 0 & -1+c^{2} \\
1 & 0 & 1-c^{2} & 0 \\
0 & 1 & 0 & 1 \\
-1 & 0 & -1 & 0
\end{array}\right) .
$$

Furthermore the hyper-Kähler structure $\left(h, \omega_{1}, \omega_{2}, \omega_{3}\right)$ induced on $T^{*} \mathbb{R}^{2}$ by the coordinate system $\mathbf{X}$ is given, in terms of the coordinate system $\{x, y, p, q\}$, by

$$
\begin{aligned}
& \omega_{1}=\mathrm{d} x \wedge \mathrm{d} y+\mathrm{d} p \wedge \mathrm{d} y+\mathrm{d} x \wedge \mathrm{d} q+\left(1-c^{2}\right) \mathrm{d} p \wedge \mathrm{d} q \\
& \omega_{2}=c(\mathrm{~d} x \wedge \mathrm{d} q+\mathrm{d} p \wedge \mathrm{d} y+2 \mathrm{~d} p \wedge \mathrm{d} q) \\
& \omega_{3}=-c(\mathrm{~d} x \wedge \mathrm{d} p+\mathrm{d} y \wedge \mathrm{d} q)
\end{aligned}
$$


and the hyper-Kähler metric $h$ is given by equation (3.6). This structure ties together the geometrical and dynamical aspects of balanced models as discussed by McIntyre \& Roulstone (2002). We observe that $\omega_{2}$, whose role was unclear in McIntyre \& Roulstone (op. cit. $\S 14$ ), is the Kähler form induced by the particular choice of canonical coordinates. The holomorphic 2 -form $\omega_{1}+i \omega_{3}$ allows us to write the equation relating the potential vorticity to the geopotential as

$$
f^{-1} \zeta_{\mathrm{c}} \mathrm{d} x \wedge \mathrm{d} y=(\mathrm{d} \phi)^{*}\left(\omega_{1}+\mathrm{i} \omega_{3}\right),
$$

where the absolute constrained vorticity $\zeta_{c}$ is defined by

$$
\zeta_{c}(x, y)=f^{2} g^{-1} \phi(x, y) \mathcal{Q}_{c}(x, y),
$$

the section $\mathrm{d} \phi: \mathbb{R}^{2} \rightarrow T^{*} \mathbb{R}^{2}$ maps $(x, y)$ to $\left(x, y, \phi_{x}, \phi_{y}\right)$ and the star denotes pullback of differential forms which in this case consists in replacing $p$ and $q$ by $\phi_{x}$ and $\phi_{y}$. However, for SG, which corresponds to the case $c=0$, the coordinate system (3.5) becomes real and $I_{\omega}$ is not defined. Furthermore, we see from (3.10) and (3.11) that $\omega_{2}$ and $\omega_{3}$ vanish identically. In the following sections we show how to recover a complex structure for SG.

\section{Monge-Ampère theory}

In the previous section, following the approach of McIntyre \& Roulstone (2002) we expressed the Monge-Ampère equation relating the potential vorticity to the geopotential in terms of the hyper-Kähler structure induced by the complex coordinate system $(X, Y)$ (equation (3.12)). The purpose of this section is to invert this strategy: that is, we make the Monge-Ampère equation the object of primary interest and we apply the theory of Monge-Ampère operators developed in Lychagin (1979), Lychagin et al. (1993) and Kushner et al. (2007) to define a new hyperKähler structure. The first part of this section contains the necessary concepts to develop this approach in a second part, further detail can be found in Kushner et al. (2007).

\section{(a) Symplectic Monge-Ampère equations in $\mathbb{R}^{2}$}

A symplectic Monge-Ampère equation in $\mathbb{R}^{2}$ is a second order nonlinear differential equation of the form

$$
A \frac{\partial^{2} \phi}{\partial x^{2}}+2 B \frac{\partial^{2} \phi}{\partial x \partial y}+C \frac{\partial^{2} \phi}{\partial y^{2}}+D\left(\frac{\partial^{2} \phi}{\partial x^{2}} \frac{\partial^{2} \phi}{\partial y^{2}}-\left(\frac{\partial^{2} \phi}{\partial x \partial y}\right)^{2}\right)+E=0,
$$

where $A, B, C, D$ and $E$ are smooth functions on the cotangent bundle $T^{*} \mathbb{R}^{2}$. We endow $T^{*} \mathbb{R}^{2}$ with the coordinate system $\{x, y, p, q\}$ and we denote by $\Omega \in \Omega^{2}\left(T^{*} \mathbb{R}^{2}\right)$ the canonical symplectic form on $T^{*} \mathbb{R}^{2}$ given by

$$
\Omega=\mathrm{d} x \wedge \mathrm{d} p+\mathrm{d} y \wedge \mathrm{d} q .
$$

A 2-form $\omega \in \Omega^{2}\left(T^{*} \mathbb{R}^{2}\right)$ is said to be effective if $\omega \wedge \Omega=0$. The Hodge-LepageLychagin theorem (Lychagin (1979)) shows that there is a one-to-one correspondence between effective 2 -forms on $T^{*} \mathbb{R}^{2}$ and symplectic Monge-Ampère equations in $\mathbb{R}^{2}$ given by the map

$$
\omega \mapsto \Delta_{\omega} \phi=0,
$$


where for any smooth function $\phi$ on $\mathbb{R}^{2}$, the Monge-Ampère operator $\Delta_{\omega}$ is defined by

$$
\Delta_{\omega} \phi=(\mathrm{d} \phi)^{*} \omega .
$$

As previously $\mathrm{d} \phi: \mathbb{R}^{2} \rightarrow T^{*} \mathbb{R}^{2}$ is the section defined by $(x, y) \mapsto\left(x, y, \phi_{x}, \phi_{y}\right)$ and the star denotes the pull-back of differential forms, which, again, consists in replacing $p$ and $q$ by $\phi_{x}$ and $\phi_{y}$ in this case. We denote the Monge-Ampère equation $\Delta_{\omega} \phi=0$ by $\mathcal{E}_{\omega}$. A smooth function $\phi: \mathbb{R}^{2} \rightarrow \mathbb{R}$ such that $\Delta_{\omega} \phi=0$ is called a classical solution of $\mathcal{E}_{\omega}$. A generalized (or multivalued) solution of $\mathcal{E}_{\omega}$ is a submanifold $L \subset T^{*} \mathbb{R}^{2}$ such that $L$ is bilagrangian with respect to $(\Omega, \omega)$, that is $\left.\Omega\right|_{L}=0$ and $\left.\omega\right|_{L}=0$. The latter definition is compatible with the notion of classical solution; indeed if $\phi$ is a classical solution of the equation $\mathcal{E}_{\omega}$ then the graph of $\mathrm{d} \phi$ defines a generalized solution of $\mathcal{E}_{\omega}$. Conversely a generalized solution $L$ is locally the graph of a classical solution $\phi$ if the projection $L \rightarrow \mathbb{R}^{2}$ is a local diffeomorphism. Two symplectic Monge-Ampère equations $\mathcal{E}_{\omega_{1}}$ and $\mathcal{E}_{\omega_{2}}$ are said to be symplectically equivalent if there exists a symplectomorphism $F: T^{*} \mathbb{R}^{2} \rightarrow T^{*} \mathbb{R}^{2}$ such that

$$
F^{*} \omega_{1}=\omega_{2}
$$

and in this case, if $L$ is a generalized solution of $\mathcal{E}_{F^{*} \omega_{1}}=\mathcal{E}_{\omega_{2}}$ then $F(L)$ is a generalized solution of $\mathcal{E}_{\omega_{1}}$.

To any effective 2 -form $\omega$ on $T^{*} \mathbb{R}^{2}$, and to any symplectic Monge-Ampère equation $\mathcal{E}_{\omega}$, we associate the field of endomorphisms $A_{\omega}: T^{*} \mathbb{R}^{2} \rightarrow \operatorname{End}\left(T^{*} \mathbb{R}^{2}\right)$ defined by $\omega(\cdot, \cdot)=\Omega\left(A_{\omega} \cdot, \cdot\right)$. As shown in Kushner et al. (2007 $\left.\S 6.1\right)$, when $\omega$ is effective we have

$$
A_{\omega}^{2}+\operatorname{pf}(\omega)=0
$$

where $\operatorname{pf}(\omega)$ is the scalar, called the pfaffian of $\omega$, defined by

$$
\omega \wedge \omega=\operatorname{pf}(\omega) \Omega \wedge \Omega .
$$

For any effective 2 -form $\omega$, the equation $\mathcal{E}_{\omega}$ is said to be: nondegenerate if and only if $\operatorname{pf}(\omega) \neq 0$, elliptic if $\mathrm{pf}(\omega)>0$ and hyperbolic if $\mathrm{pf}(\omega)<0$. For reasons that we make explicit in the following subsection, we restrict ourselves to the study of elliptic equations. From equation (4.3) we then see that the field of endomorphisms $I_{\omega}$ given by

$$
I_{\omega}=|\operatorname{pf}(\omega)|^{-1 / 2} A_{\omega},
$$

defines an almost-complex structure structure on $T^{*} \mathbb{R}^{2}$, and any generalized solution of $\mathcal{E}_{\omega}$ defines a $I_{\omega}$-holomorphic curve. Finally, we have the following result due to Lychagin et al. (1993), and summarised by Banos (2006) as follows:

Theorem 4.1. Let $\mathcal{E}_{\omega}$ be a symplectic Monge-Ampère equation on $\mathbb{R}^{2}$, then the following assertions are equivalent:

1. $\mathcal{E}_{\omega}$ is symplectically equivalent to the equation

- $\phi_{x x}+\phi_{y y}=0$ when $\operatorname{pf}(\omega)>0$,

- $\phi_{x x}-\phi_{y y}=0$ when $\operatorname{pf}(\omega)<0$.

2. The structure $I_{\omega}$ is integrable.

3. The normalized 2-form $|\mathrm{pf}(\omega)|^{-1 / 2} \omega$ is closed.

We now investigate SG theory and HBMs, regarding the Monge-Ampère equation as the primary object of interest. 
(b) Hyper-Kähler geometry 86 balanced models: Monge-Ampère operators

Using the absolute constrained vorticity $\zeta_{c}$ defined by equation (3.13), the equation relating the potential vorticity $\mathcal{Q}_{\mathrm{c}}$ to the geopotential $\phi$ becomes a symplectic Monge-Ampère equation for $\phi$ given $\zeta_{c}$ which can be written as

$$
0=\left(1-f^{-1} \zeta_{c}\right)+\frac{\partial^{2} \phi}{\partial x^{2}}+\frac{\partial^{2} \phi}{\partial y^{2}}+\left(1-c^{2}\right)\left(\frac{\partial^{2} \phi}{\partial x^{2}} \frac{\partial^{2} \phi}{\partial y^{2}}-\left(\frac{\partial^{2} \phi}{\partial x \partial y}\right)^{2}\right)
$$

The corresponding effective 2-form $\omega \in \Omega_{\epsilon}^{2}\left(T^{*} \mathbb{R}^{2}\right)$ is the closed 2-form given by

$$
\omega=\left(1-f^{-1} \zeta_{\mathrm{c}}\right) \mathrm{d} x \wedge \mathrm{d} y+\mathrm{d} p \wedge \mathrm{d} y+\mathrm{d} x \wedge \mathrm{d} q+\left(1-c^{2}\right) \mathrm{d} p \wedge \mathrm{d} q
$$

whose pfaffian is given by

$$
\operatorname{pf}(\omega)=1-\left(1-c^{2}\right)\left(1-f^{-1} \zeta_{c}\right)
$$

Recall that $c$ is a real parameter taking certain specific values (in the range $0 \leq c \leq$ $\sqrt{3})$; moreover the assumptions under which balanced models are valid are $\epsilon \ll 1$ and $f^{-1} \zeta_{c}=1+\mathcal{O}(\epsilon)$. Therefore we have $\mathrm{pf}(\omega)>0$ for the physical regimes of interest, then equation (4.6) is elliptic. The almost-complex structure $I_{\omega}$ is given by

$$
I_{\omega}=\frac{1}{\sqrt{\mathrm{pf}(\omega)}}\left(\begin{array}{cccc}
0 & -1 & 0 & -1+c^{2} \\
1 & 0 & 1-c^{2} & 0 \\
0 & 1-f^{-1} \zeta_{\mathrm{c}} & 0 & 1 \\
-1+f^{-1} \zeta_{\mathrm{c}} & 0 & -1 & 0
\end{array}\right) .
$$

From Theorem 4.1 we see that $I_{\omega}$ is integrable if and only if $\zeta_{c}$ is constant in space. Although this condition is very restrictive for practical use, many important idealized features of SG have been studied in the context of constant coefficient problems (e.g. Chynoweth \& Sewell (1989), Hoskins \& West (1979), Shutts (1991); see also Roulstone et al. (2009a) and McIntyre \& Roulstone (2002), §14).

Restricting ourselves to the case where $\zeta_{c}$ is a constant denoted by $\zeta_{0}$, we seek an adapted coordinate system for $I_{\omega}$ to associate a Kähler structure with the MongeAmpère equation (4.6). Recall that in the preceding section, the coordinate system was specified and the structures were derived by considering the standard complex metric (3.2). In the present case there is a priori no reason to favour any metric among the set of all Hermitian metrics with respect to $I_{\omega}$. Noting (3.5), we choose to work with the complex coordinate system $\left(X_{2}, Y_{2}\right)$ defined on $T^{*} \mathbb{R}^{2}$ by

$$
\begin{aligned}
& X_{2}=\alpha_{1} x+\mathrm{i} \alpha_{2} y+\beta_{1} p+\mathrm{i} \beta_{2} q, \\
& Y_{2}=\alpha_{1} y-\mathrm{i} \alpha_{2} x+\beta_{1} q-\mathrm{i} \beta_{2} p,
\end{aligned}
$$

with $\left(\alpha_{1}, \alpha_{2}\right),\left(\beta_{1}, \beta_{2}\right) \in \mathbb{R}^{2}-\{(0,0)\}$. We use the subscript 2 because in our construction of the hyper-Kähler structure $\left(h, \omega_{1}, \omega_{2}, \omega_{3}\right)$ induced by a complex coordinate system $(X, Y)$ and defined by equations (3.2) and (3.4), the coordinate system is an adapted coordinate system for the complex structure $I_{2}$. The subscript ${ }_{2}$ in the coordinate system $\left(X_{2}, Y_{2}\right)$ defined above is to remind us of this fact. This notation will prove useful in the following section where we will need to distinguish between the three different identifications of $T^{*} \mathbb{R}^{2}$ as $\mathbb{C}^{2}$ induced by the three 
Kähler forms $\omega_{1}, \omega_{2}$ and $\omega_{3}$. Inserting equation (4.10) in equation (3.2) we see that the hyper-Kähler metric induced by $\left(X_{2}, Y_{2}\right)$ is given by

$$
\begin{aligned}
h= & \left(\alpha_{1}^{2}+\alpha_{2}^{2}\right)(\mathrm{d} x \otimes \mathrm{d} x+\mathrm{d} y \otimes \mathrm{d} y)+\left(\alpha_{1} \beta_{1}+\alpha_{2} \beta_{2}\right)(\mathrm{d} x \otimes \mathrm{d} p+\mathrm{d} y \otimes \mathrm{d} q) \\
& +\left(\beta_{1}^{2}+\beta_{2}^{2}\right)(\mathrm{d} p \otimes \mathrm{d} p+\mathrm{d} q \otimes \mathrm{d} q),
\end{aligned}
$$

and inserting (4.10) in (3.4), we obtain the triple of Kähler forms

$$
\begin{aligned}
& \omega_{1}=\left(\alpha_{1}^{2}-\alpha_{2}^{2}\right) \mathrm{d} x \wedge \mathrm{d} y+\left(\alpha_{1} \beta_{1}-\alpha_{2} \beta_{2}\right)(\mathrm{d} x \wedge \mathrm{d} q+\mathrm{d} p \wedge \mathrm{d} y)+\left(\beta_{1}^{2}-\beta_{2}^{2}\right) \mathrm{d} p \wedge \mathrm{d} q \\
& \omega_{2}=2 \alpha_{1} \alpha_{2} \mathrm{~d} x \wedge \mathrm{d} y+\left(\alpha_{1} \beta_{2}+\alpha_{2} \beta_{1}\right)(\mathrm{d} x \wedge \mathrm{d} q+\mathrm{d} p \wedge \mathrm{d} y)+2 \beta_{1} \beta_{2} \mathrm{~d} p \wedge \mathrm{d} q, \\
& \omega_{3}=-\left(\alpha_{1} \beta_{2}-\alpha_{2} \beta_{1}\right)(\mathrm{d} x \wedge \mathrm{d} p+\mathrm{d} y \wedge \mathrm{d} q) .
\end{aligned}
$$

We identify the 2-form $\omega_{1}$ with the effective 2 -form (4.7), therefore we are led to consider the underdetermined system of equations for $\alpha_{1}, \alpha_{2}, \beta_{1}, \beta_{2}$

$$
\left\{\begin{array}{cl}
1-f^{-1} \zeta_{0} & =\alpha_{1}^{2}-\alpha_{2}^{2}, \\
1 & =\alpha_{1} \beta_{1}-\alpha_{2} \beta_{2}, \\
1-c^{2} & =\beta_{1}^{2}-\beta_{2}^{2} .
\end{array}\right.
$$

By considering $\gamma=\alpha_{2}$ as a parameter and inverting (4.11) with the assumption that $1-f^{-1} \zeta_{0}+\gamma^{2}>0$, and taking the positive roots, we can write

$$
\begin{array}{ll}
\alpha_{1}=\sqrt{1-f^{-1} \zeta_{0}+\gamma^{2}} \quad, & \beta_{1}=\frac{\sqrt{1-f^{-1} \zeta_{0}+\gamma^{2}}+\gamma \sqrt{\mathrm{pf}(\omega)}}{1-f^{-1} \zeta_{0}}, \\
\alpha_{2}=\gamma, & \beta_{2}=\frac{\gamma+\sqrt{1-f^{-1} \zeta_{0}+\gamma^{2}} \sqrt{\mathrm{pf}(\omega)}}{1-f^{-1} \zeta_{0}}
\end{array}
$$

(providing $1-f^{-1} \zeta_{0} \neq 0$ ). Setting $\gamma=0$, from (4.10) we obtain the coordinate system

$$
\begin{aligned}
& X_{2}=\sqrt{1-f^{-1} \zeta_{0}} x+\frac{1}{\sqrt{1-f^{-1} \zeta_{0}}} p+\mathrm{i} \sqrt{\frac{\mathrm{pf}(\omega)}{1-f^{-1} \zeta_{0}}} q, \\
& Y_{2}=\sqrt{1-f^{-1} \zeta_{0}} y+\frac{1}{\sqrt{1-f^{-1} \zeta_{0}}} q-\mathrm{i} \sqrt{\frac{\mathrm{pf}(\omega)}{1-f^{-1} \zeta_{0}}} p,
\end{aligned}
$$

where $\mathrm{pf}(\omega)$ is given by (4.8). The hyper-Kähler structure $\left(h, \omega_{1}, \omega_{2}, \omega_{3}\right)$ induced by $\left(X_{2}, Y_{2}\right)$, defined by $(4.13)$, is given by

$$
\begin{aligned}
h= & \left(1-f^{-1} \zeta_{0}\right)(\mathrm{d} x \otimes \mathrm{d} x+\mathrm{d} y \otimes \mathrm{d} y)+\mathrm{d} x \otimes \mathrm{d} p+\mathrm{d} y \otimes \mathrm{d} q \\
& +\frac{1+\mathrm{pf}(\omega)}{1-f^{-1} \zeta_{0}}(\mathrm{~d} p \otimes \mathrm{d} p+\mathrm{d} q \otimes \mathrm{d} q) \\
\omega_{1}= & \left(1-f^{-1} \zeta_{0}\right) \mathrm{d} x \wedge \mathrm{d} y+\mathrm{d} p \wedge \mathrm{d} y+\mathrm{d} x \wedge \mathrm{d} q+\left(1-c^{2}\right) \mathrm{d} p \wedge \mathrm{d} q \\
\omega_{2}= & \sqrt{\operatorname{pf}(\omega)}\left(\mathrm{d} x \wedge \mathrm{d} q+\mathrm{d} p \wedge \mathrm{d} y+\frac{2}{1-f^{-1} \zeta_{0}} \mathrm{~d} p \wedge \mathrm{d} q\right) \\
\omega_{3}= & -\sqrt{\operatorname{pf}(\omega)}(\mathrm{d} x \wedge \mathrm{d} p+\mathrm{d} y \wedge \mathrm{d} q) .
\end{aligned}
$$

When $c=0$, noting from (4.8) that $\operatorname{pf}(\omega)=f^{-1} \zeta_{0}$, we obtain a new hyper-Kähler structure for SG theory. Note (see also McIntyre \& Roulstone (2002), §14) that the term $1-f^{-1} \zeta_{0}$ is not sign-definite, it is a dimensionless measure of minus the relative vorticity: when $1-f^{-1} \zeta_{0}>0$ the dynamics is cyclonic, when $1-f^{-1} \zeta_{0}<0$ the dynamics is anticyclonic and $\sqrt{1-f^{-1} \zeta_{0}}$ is complex. 


\section{Legendre structure}

Having found a hyper-Kähler structure for SG, we now ask if the Legendre duality of the geostrophic momentum coordinates (2.5) can be generalized to the new complex coordinates.

In $\S 2 . b$ we presented the holomorphic potential $\Phi$, given by equation $(2.16)$ as a function of the coordinates $X$ and $\bar{Y}$ defined using equation (2.14). Using this complex potential Roulstone \& Roubtsov (2001) exhibited contact properties for HBMs. The hyper-Kähler structure $\left(\tilde{h}, \tilde{\omega}_{1}, \tilde{\omega}_{2}, \tilde{\omega}_{3}\right)$ induced by the coordinate system $(X, \bar{Y})$ is given by

$$
\tilde{h}=\mathrm{d} x \otimes \mathrm{d} x+\mathrm{d} y \otimes \mathrm{d} y+\mathrm{d} x \otimes \mathrm{d} p+\mathrm{d} y \otimes \mathrm{d} q+\left(1+c^{2}\right)(\mathrm{d} p \otimes \mathrm{d} p+\mathrm{d} q \otimes \mathrm{d} q),
$$

and

$$
\begin{aligned}
& \tilde{\omega}_{1}=\mathrm{d} x \wedge \mathrm{d} y+\mathrm{d} p \wedge \mathrm{d} y+\mathrm{d} x \wedge \mathrm{d} q+\left(1+c^{2}\right) \mathrm{d} p \wedge \mathrm{d} q, \\
& \tilde{\omega}_{2}=-c(\mathrm{~d} x \wedge \mathrm{d} q-\mathrm{d} p \wedge \mathrm{d} y), \\
& \tilde{\omega}_{3}=c(\mathrm{~d} x \wedge \mathrm{d} p-\mathrm{d} y \wedge \mathrm{d} q) .
\end{aligned}
$$

This structure differs from the hyper-Kähler structure $\left(h, \omega_{1}, \omega_{2}, \omega_{3}\right)$ induced by the coordinate system $(X, Y)$ given by (3.6) and (3.9)-(3.11), and no longer corresponds to the dynamical properties, nor the geometrical properties, of HBMs (essentially, this aforementioned structure corresponds to a hyperbolic Monge-Ampère equation). In this section, by considering the hyper-Kähler structure induced by the coordinate system $\left(X_{2}, Y_{2}\right)$ given by $(4.10)$, we shall extend the Legendre duality of SG theory and define a holomorphic potential from which the contact properties of HBMs will be derived.

Let $A$ and $B$ be $2 \times 2$ nondegenerate commuting symmetric complex matrices. We define the complex coordinates $\mathbf{X}=(X, Y)^{\mathrm{T}}$ by

$$
\mathbf{X}=A \mathbf{x}+B \mathbf{p}
$$

with $\mathbf{p}=(p, q)^{\mathrm{T}}=\left(\partial_{x} \phi, \partial_{y} \phi\right)^{\mathrm{T}}$. Let us define $\mathcal{P}(\mathbf{x}), \mathcal{R}(\mathbf{X})$ and $\Phi(\mathbf{X})$ by

$$
\begin{aligned}
\mathcal{P}(\mathbf{x}) & =\phi(\mathbf{x})+\frac{1}{2} \mathbf{x}^{\mathrm{T}} B^{-1} A \mathbf{x} \\
\mathcal{R}(\mathbf{X}) & =\mathbf{X}^{\mathrm{T}} B^{-1} \mathbf{x}-\mathcal{P} \\
\Phi(\mathbf{X}) & =-\mathcal{R}+\frac{1}{2} \mathbf{X}^{\mathrm{T}} B^{-1} A^{-1} \mathbf{X} .
\end{aligned}
$$

We have

$$
\nabla_{\mathbf{x}} \mathcal{P}=B^{-1} \mathbf{X}, \nabla_{\mathbf{X}} \mathcal{R}=B^{-1} \mathbf{x}
$$

where $\nabla_{\mathbf{x}}=(\partial / \partial x, \partial / \partial y)^{\mathrm{T}}$ and $\nabla_{\mathbf{X}}=(\partial / \partial X, \partial / \partial Y)^{\mathrm{T}}$. We obtain the gradient property

$$
\nabla_{\mathbf{X}} \Phi=A^{-1} \nabla_{\mathbf{x}} \phi
$$

Restricting ourselves to the case where $A$ or $B$ is of the form $a \mathbf{1}_{2}, a \in \mathbb{C}$, we can show that

$$
\frac{\partial \Phi}{\partial \bar{X}}=0, \frac{\partial \Phi}{\partial \bar{Y}}=0
$$


that is, $\Phi$ is a holomorphic function of $X$ and $Y$. Consider the semi-holomorphic contact bundle corresponding to the coordinates $(X, Y)$, that is, the space spanned by the coordinate system $(X, Y, \Phi, P, Q)$ endowed with the contact form $\mathrm{d} \Phi-P \mathrm{~d} X-$ $Q \mathrm{~d} Y$, with $(P, Q)^{\mathrm{T}}=(\partial \Phi / \partial X, \partial \Phi / \partial Y)^{\mathrm{T}}$; we find that if $\Phi$ satisfies (5.7), then the transformation $(x, y, \phi, p, q) \mapsto(X, Y, \Phi, P, Q)$ is a strict contact transformation.

The coordinate system $\left(X_{2}, \bar{Y}_{2}\right)$ obtained from equation (4.10) is of the form of equation (5.1) and then we can derive a Legendre transformation using (5.2)(5.4). However, as with the coordinate system $(X, \bar{Y})$ given by $(2.14)$ discussed at the beginning of this section, the hyper-Kähler structure induced by $\left(X_{2}, \bar{Y}_{2}\right)$ does not correspond to the elliptic Monge-Ampère structures of HBMs. Nevertheless, we can obtain the appropriate structure by starting with the hyper-Kähler structure $\left(h, \omega_{1}, \omega_{2}, \omega_{3}\right)$ induced by the general coordinate system $\left(X_{2}, Y_{2}\right)$. This hyper-Kähler structure is, by construction, expressed relative to the complex structure $I_{2}$. The following proposition provides us with formulae for adapted coordinate systems $\left(X_{i}, Y_{i}\right), i=1,2,3$, relative to the complex structures $I_{i}$ respectively, and inducing the same hyper-Kähler structure, as we require.

Proposition 5.1. Let $\left(h, I_{1}, I_{2}, I_{3}\right)$ be the canonical hyper-Kähler structure induced by the coordinate system $\left(X_{2}, Y_{2}\right)$ defined by (4.10). Then by construction $\left(X_{2}, Y_{2}\right)$ is an adapted coordinate system relative to $I_{2}$, and adapted coordinate systems $\left(X_{1}, Y_{1}\right),\left(X_{3}, Y_{3}\right)$ relative to $I_{1}$ and $I_{3}$ are given by

$$
\begin{gathered}
\left\{\begin{array}{c}
X_{1}=\alpha_{1}(x-\mathrm{i} y)+\beta_{1}(p-\mathrm{i} q) \\
Y_{1}=\alpha_{2}(x+\mathrm{i} y)+\beta_{2}(p+\mathrm{i} q)
\end{array}\right. \\
\left\{\begin{array}{c}
X_{3}=\left(\alpha_{1}+\mathrm{i} \alpha_{2}\right) x+\left(\beta_{1}+\mathrm{i} \beta_{2}\right) p \\
Y_{3}=\left(\alpha_{1}+\mathrm{i} \alpha_{2}\right) y+\left(\beta_{1}+\mathrm{i} \beta_{2}\right) q
\end{array}\right.
\end{gathered}
$$

This result is proved in Delahaies (2009) by showing that $I_{i}^{*} \mathrm{~d} \mathbf{X}_{i}=\mathrm{id} \mathbf{X}_{i}$, $I_{i}^{*} \mathrm{~d} \overline{\mathbf{X}}_{1}=-\mathrm{id} \overline{\mathbf{X}}_{i}$, for $i=1,3$, where $\mathbf{X}_{i}=\left(X_{i}, Y_{i}\right)^{\mathrm{T}}$. This proposition provides us with two new points of view to look at the same geometric structure.

We see that the coordinate system $\mathbf{X}_{3}$ is of the form (5.1) with

$$
A=\left(\alpha_{1}+\mathrm{i} \alpha_{2}\right) \mathbf{1}_{2}, B=\left(\beta_{1}+\mathrm{i} \beta_{2}\right) \mathbf{1}_{2},
$$

we can therefore build a Legendre structure and show contact properties using formulae (5.2)-(5.4), and by construction the hyper-Kähler structure induced by $\mathbf{X}_{3}$ is the same as the hyper-Kähler structure induced by $\mathbf{X}_{2}$. In particular with the choice of parameter (4.11) with $\gamma=0$ we have

$$
\begin{aligned}
& X_{3}=\sqrt{1-f^{-1} \zeta_{0}} x+\frac{1+\mathrm{i} \sqrt{\mathrm{pf}(\omega)}}{\sqrt{1-f^{-1} \zeta_{0}}} p, \\
& Y_{3}=\sqrt{1-f^{-1} \zeta_{0}} y+\frac{1+\mathrm{i} \sqrt{\mathrm{pf}(\omega)}}{\sqrt{1-f^{-1} \zeta_{0}}} q .
\end{aligned}
$$

and the construction above gives

$$
\mathcal{P}(x, y)=\phi+\frac{1}{2} \frac{1-f^{-1} \zeta_{0}}{1+\mathrm{i} \sqrt{\operatorname{pf}(\omega)}}\left(x^{2}+y^{2}\right),
$$




$$
\begin{gathered}
\mathcal{R}\left(X_{3}, Y_{3}\right)=\frac{\sqrt{1-f^{-1} \zeta_{0}}}{1+\mathrm{i} \sqrt{\mathrm{pf}(\omega)}}\left(x X_{3}+y Y_{3}\right)-\mathcal{P}, \\
\Phi\left(X_{3}, Y_{3}\right)=-\mathcal{R}+\frac{1}{2} \frac{1}{1+\mathrm{i} \sqrt{\mathrm{pf}(\omega)}}\left(X_{3}^{2}+Y_{3}^{2}\right) .
\end{gathered}
$$

These formulae generalize the Legendre duality of SG theory to the class of balanced models derived by McIntyre \& Roulstone, in particular for $L_{1}$-dynamics and the $\sqrt{3}$-model. For SG theory, for which $c=0$ and then $\operatorname{pf}(\omega)=f^{-1} \zeta_{0}$, the Legendre dual functions given by (2.7) and (2.8), the Bernoulli potential given by (2.10) and the geostrophic coordinates (2.5) are recovered by setting $\zeta_{0}=0$ in $(5.11)-(5.15)$.

\section{Concluding remarks}

We have revisited the issues raised in McIntyre \& Roulstone (2002) about hyperKähler structures and HBMs. In particular, in $\S 14$ of McIntyre \& Roulstone (op. cit.) three issues were raised: $i$ ) the role of one of the members of the hyper-Kähler triple of forms was unclear - ostensibly not corresponding to either a symplectic or a Monge-Ampère structure; this has now been clarified by (3.10) et seq.. ii) The signdefiniteness of $1-f^{-1} \zeta_{c}$ implied that the Kähler structure could only be exhibited for cyclonic flows; this conundrum has now been resolved by the approach adopted in $\S 4$. iii) Integrability conditions restrict our results to consideration of constant coefficient Monge-Ampère equations. While this remains the case, it is perhaps not surprising that anything but the simplest idealized structures in geophysical flows should possess these highly abstract geometrical properties. Similar issues have been addressed in the context of incompressible Navier-Stokes flows in two and three dimensions (see Roulstone et al. (2009a, 2009b)).

In summary, using the formalism of Kushner et al. (2007), we have exhibited a new hyper-Kähler structure for HBMs, which incorporates SG theory and, using the properties of the hyper-Kähler geometry, we show how Legendre duality is exhibited in other HBMs.

An over-arching question remains: what is the significance of these complex structures in fluid mechanics, and how might the geometry enhance our understanding of balanced flows and/or turbulence? Although it would be premature to attempt to give an unequivocal answer, Roulstone et al. (2009) have shown how almost-complex structures can be used to describe coherent structures in NavierStokes flows in three dimensions. The salient point of this work is that complex geometry provides a framework for studying coherent structures that is not readily accessible via the more traditional analysis of the underlying partial differential equations that can be performed for incompressible flows in two dimensions (e.g. Larchevêque (1993)). The results of Roulstone et al. op. cit. are based on the work of Banos (2002) on Monge-Ampere structures for three independent variables. It is tempting to speculate that Banos' results could be applied to SG in three dimensions, but progress in this direction requires an understanding of how the complex geometry associated with Monge-Ampère structures can be applied to SG in two dimensions. In this paper, we have laid the foundations for such developments.

Acknowledgements We would like to thank Professor V.N Roubtsov for useful discussions. The work of S. Delahaies was supported, in part, by a NERC studentship. 


\section{References}

Allen, J.S \& Holm, D.D. 1996 Extended-geostrophic Hamiltonian models for rotating shallow water motion. Physica D 98, 229-48.

Banos, B. 2006 Monge-Ampere equations and generalized complex geometry. The twodimensional case. Available at http://arxiv.org/abs/math/0603432.

Blumen, W. 1981 The geostrophic coordinate transformation. J. Atmos. Sc. 38, 11001105.

Chynoweth, S. \& Sewell, M.J. 1989 Dual Variables in Semigeostrophic Theory. Proc. Roy. Soc. Lond. A 424, 155-186.

Cullen, M.J.P. \& Purser, R.J. 1984 An extended Lagrangian theory of semi-geostrophic frontogenesis. J. Atmos. Sci. 41, 1477-1497.

Cullen, M.J.P. \& Purser,R.J. 1989 Properties of the Lagrangian semi-geostrophic equations. J. Atmos. Sci. 46, 2684-2697.

Delahaies, S. 2009 Complex and contact geometry in geophysical fluid dynamics. PhD Thesis, University of Surrey, U.K.

Hoskins, B.J. 1975 The geostrophic momentum approximation and the semi-geostrophic equations. J. Atmos. Sci. 32, 233-242.

Hoskins, B.J., West N.V. 1979 Baroclinic Waves and Frontogenesis. Part II: Uniform Potential Vorticity Jet Flows-Cold and Warm Fronts. J. Atmos. Sci, 36, 1663-1680.

Kushner, A., Lychagin, V., Rubtsov, V. 2007 Contact Geometry and Nonlinear Differential Equations. Cambridge University Press.

Larchevêque, M. 1993 Pressure field, vorticity field, and coherent structures in twodimensional incompressible turbulent flows. Theor. Comp. Fluid Dynamics, 5, 215-222.

Lychagin, V.V. 1979 Contact geometry and non-linear second order differential equations. Russian Math. Surveys, 34.

Lychagin, V.V., Roubtsov, V., Chekalov, I. 1993 A classification of Monge Ampère equations. Ann. scien. Ec. Norm. Sup., 4ème série, 26, 281-308.

McDuff, D. \& Salamon, D. 1998 Introduction to Symplectic Topology. Oxford University Press.

McIntyre, M.E. \& Roulstone, I. 1996 Hamiltonian balanced models: slow manifolds, constraints and velocity splitting. Forecasting Research Scientific Paper 41, Meteorological Office, U.K.

McIntyre, M.E. \& Roulstone, I. 2001 Are there higher-accuracy analogues of semigeostrophic theory? In Large-Scale Atmosphere-Ocean Dynamics, vol II, Geometric methods and models (ed. I. Roulstone \& J. Norbury). Cambridge University Press.

Roubtsov, V. \& Roulstone, I. 1997 Examples of quaternionic and kahler structures in hamiltonian models of nearly geostrophic flow. J. Phys. A: Math. Gen., 30,63-68.

Roubtsov, V. \& Roulstone, I. 2001 Holomorphic structures in hydrodynamical models of nearly geostrophic flow. Proc. R. Soc. Lond. A 457, 1519-1531.

Roulstone, I., Banos, B., Gibbon, J.D. \& Roubtsov, V.N 2009a Kähler geometry and Burgers' vortices. To appear in Proc. Math. Inst. Natl. Acad. Sci. Ukraine, in honour of the 60 th birthday of I. S. Krasil'schik.

Roulstone, I., Banos, B., Gibbon, J.D. \& Roubtsov, V.N 2009b A geometric interpretation of coherent structures in Navier-Stokes flows. To appear in Proc. R. Soc. Lond. A.

Roulstone, I. \& Sewell, M.J. 1997 The mathematical structure of theories of semigeostrophic type. Phil. Trans. R. Soc. Lond. A 355, 2489-2517.

Salmon, R. 1983 Practical use of Hamilton's principle. J. Fluid Mech. 132, 431-444.

Salmon, R. 1985 New equations for nearly geostrophic flow. J. Fluid Mech. 153, 461-477.

Shutts, G. 1991 Some Exact Solutions to Semi-Geosgrophic Equations for Uniform Potential Vorticity Flows. Geophysical \& Astrophysical Fluid Dynamics, 57:99-114. 
Snyder, C., Skamarock, W.C., Rotunno, R. 1991 A Comparison of Primitive-Equation and Semigeostrophic Simulations of Baroclinic Waves. J. Atmos. Sci., 46:2179-2194. 